\title{
Heating and Turbulence Driving by Galaxy Motions in Galaxy Clusters
}

\author{
Woong-Tae Kim \\ Department of Physics \& Astronomy, FPRD, Seoul National University, Seoul 151-742, \\ Republic of Korea \\ wkim@astro.snu.ac.kr
}

\begin{abstract}
Using three-dimensional hydrodynamic simulations, we investigate heating and turbulence driving in an intracluster medium (ICM) by orbital motions of galaxies in a galaxy cluster. We consider $N_{g}$ member galaxies on isothermal and isotropic orbits through an ICM typical of rich clusters. An introduction of the galaxies immediately produces gravitational wakes, providing perturbations that can potentially grow via resonant interaction with the background gas. When $N_{g}^{1 / 2} M_{11} \lesssim 100$, where $M_{11}$ is each galaxy mass in units of $10^{11} \mathrm{M}_{\odot}$, the perturbations are in the linear regime and the resonant excitation of gravity waves is efficient to generate kinetic energy in the ICM, resulting in the velocity dispersion $\sigma_{v} \sim 2.2 N_{g}^{1 / 2} M_{11} \mathrm{~km} \mathrm{~s}^{-1}$. When $N_{g}^{1 / 2} M_{11} \gtrsim 100$, on the other hand, nonlinear fluctuations of the background ICM destroy galaxy wakes and thus render resonant excitation weak or absent. In this case, the kinetic energy saturates at the level corresponding to $\sigma_{v} \sim 220 \mathrm{~km} \mathrm{~s}^{-1}$. The angle-averaged velocity power spectra of turbulence driven in our models have slopes in the range of -3.7 to -4.3 . With the nonlinear saturation of resonant excitation, none of the cooling models considered are able to halt cooling catastrophe, suggesting that the galaxy motions alone are unlikely to solve the cooling flow problem.
\end{abstract}

Subject headings: cooling flows — galaxies: clusters : general — turbulence — waves - X-rays: galaxies

\section{INTRODUCTION}

A lack of cold gas in the central parts of rich galaxy clusters, as revealed by highresolution X-ray observations, has posed a "cooling flow" problem, requiring sources of heat 
to balance radiative cooling of an intracluster medium (ICM). Among the proposed heating mechanisms (see Peterson \& Fabian 2006 for review), energy injection from active galactic nuclei appears to be the most favorable, although it requires a central black hole to be more massive than observed (Fujita \& Reiprich 2004) and is unable to maintain a long term energy balance if jets are narrow (Vernaleo \& Reynolds 2006). Diffusive heating via thermal conduction and/or turbulent mixing may also be effective if the relevant diffusion coefficient is quite large and fine tuned (e.g., Kim \& Naravan 2003; Voigt \& Fabian 2004; Dennis \& Chandran 2005).

Less well recognized is the ICM heating by cluster galaxies that possess a lot of available energy in their motions (e.g., Miller 1986; Bregman \& David 1989). Balbus \& Soker (1990, hereafter BS) showed that resonant excitations of internal waves driven by orbiting galaxies produce heat at cluster centers comparable to radiative loss, provided the galaxy mass is large enough. Using Monte-Carlo approaches, El-Zant et al (2004) showed that dynamical friction of galaxies can be a distributed source of heat if the mass-to-light ratio of galaxies

exceeds 10. Kim et al (2005) found that heating by dynamical friction reduces the growth rates of thermal instability. All these works suggest that the effects of galaxy motions on thermodynamic evolution of ICM are by no means negligible.

Most of the studies cited above are based on the assumption that the energy lost by galaxies is all transferred to thermal energy of the ICM locally near the galaxies. This is apparently not the case because galaxy wakes are spatially extended, overlap with each other, and induce kinetic as well as thermal energies (Deiss \& Just 1996; Ostriker 1999). The possibility of the ICM stirring by galaxy motions is interesting because turbulence appears to be pervasive in the ICM (e.g., Schuecker et al. 2004) and perhaps determines the characteristic strengths and scales of cluster magnetic fields (e.g., Clarke et al 2001; Subramanian et al 2006). Although BS allowed for the spatial propagation of internal waves, they focused on linear gaseous responses in the WKB limit. Lufkin et al (1995, hereafter LBH) explored nonlinear evolution of gravity waves, but their models considered a single galaxy on a radial orbit. In this Letter, we extend LHB by considering a number of cluster galaxies on isotropic orbits. By varying the mass and number of galaxies, we quantify the thermal and kinetic energies induced by galaxy motions and explore the level and shape of such driven ICM turbulence.

\section{Model and Method}

We consider a galaxy cluster in which the ICM is initially in hydrostatic equilibrium under a dark matter potential $\Phi_{\mathrm{DM}}$. For the initial temperature of the ICM, we adopt a simple 
form $T(r)=7\left[1-0.6 e^{-\left(r / r_{c}\right)^{2}}\right] \mathrm{keV}$ with the cooling radius $r_{c}=125 \mathrm{kpc}$. We represent a rigid dark halo using an NFW profile with the characteristic mass $M_{0}=6 \times 10^{14} \mathrm{M}_{\odot}$ and scale radius $r_{s}=460 \mathrm{kpc}$ (Navarro et al. 1997). Although we do not allow for the presence of a central dominant galaxy considered in LBH, the cooling core inside $r_{c}$ in our model still satisfies the condition for resonant excitation of gravity waves (see \$3.1).

To study the responses of the ICM to member galaxies, we solve the ideal hydrodynamic equations:

$$
\begin{gathered}
(\partial / \partial t+\mathbf{v} \cdot \nabla) \rho+\rho \nabla \cdot \mathbf{v}=0 \\
\rho(\partial / \partial t+\mathbf{v} \cdot \nabla) \mathbf{v}=-\nabla P-\rho \nabla\left(\Phi_{\mathrm{DM}}+\Phi_{g}\right), \\
\rho(\partial / \partial t+\mathbf{v} \cdot \nabla)(e / \rho)=-P \nabla \cdot \mathbf{v}-\Lambda,
\end{gathered}
$$

where $\Phi_{g}$ is the time-varying gravitational potential due to the galaxies and $\Lambda$ is the volumetric cooling rate. The other symbols have their usual meanings. The effects of gaseous self-gravity and magnetic fields are ignored. We adopt an ideal gas law $P=(\gamma-1) e$ with $\gamma=5 / 3$. We run both adiabatic (with $\Lambda=0$ ) and cooling (with $\Lambda \neq 0$ ) models by taking the cooling function $\Lambda$ used in Ruszkowski \& Begelman (2002).

We consider a total of $N_{g}$ member galaxies distributed within $1 \mathrm{Mpc}$. We assume that they follow isotropic and isothermal orbits with velocity dispersion $\sigma_{r}=800 \mathrm{~km} \mathrm{~s}^{-1}$, in which case the equilibrium galaxy number density is $\propto\left(1+r / r_{s}\right)^{\eta r_{s} / r}$, where $\eta \equiv 2 G M_{0} /\left(r_{s} \sigma_{r}^{2}\right) \approx$ 17.5 (Kim et al 2005). Under this distribution, about $13 \%$ of the galaxies are located within $r_{c}$ at any given time. We ignore back reaction of the ICM to the galaxies since the energy lost due to dynamical friction is small (LBH). We represent each galaxy using a Plummer potential $\Phi_{p}(r)=-G M_{g}\left(r^{2}+a^{2}\right)^{-1 / 2}$ with mass $M_{g}$ and scale radius $a=7 \mathrm{kpc}$; the total perturbing potential is constructed as $\Phi_{g}(\mathbf{r}, t)=\sum_{i=1}^{N_{g}} \Phi_{p}\left(\left|\mathbf{r}-\mathbf{r}_{i}(t)\right|\right)$, where $\mathbf{r}_{i}$ is the position vector of the $i$-th galaxy at time $t$. To simulate diverse cluster conditions, we vary the number and mass of the galaxies in the ranges of $N_{g} \sim 10^{2}-10^{3}$ and $M_{g} \sim 10^{11}-10^{12} \mathrm{M}_{\odot}$. We report in this work the results only for models where all the galaxies have equal masses 1 .

We follow the nonlinear evolution of the hot ICM using a modified version of the ZEUS code (Stone \& Norman 1992), parallelized on a distributed-memory platform. Our simulation domain is a cubic box with each side of $2 \mathrm{Mpc}$; the center of the box is located at the cluster center. We construct a logarithmically spaced Cartesian grid with $256^{3}$ zones, with

\footnotetext{
${ }^{1}$ By running a few models in which galaxy masses vary according to the Schechter function with a massto-light ratio $M / L \sim L^{0.3}$ (Gerhard et al. 2001), we have confirmed that results are almost unchanged if the breaking mass $M_{*}$ in the mass distribution is equal to $M_{g}$ in the corresponding fixed mass case.
} 
outflow conditions at all boundaries 2 . The grid spacing is $0.3,4$, and $33 \mathrm{kpc}$ at the center, the cooling radius, and the edge of the box, respectively. We have confirmed the accuracy of the code by comparing the test results for wakes produced by linear-trajectory perturbers with the analytic formula of Ostriker (1999).

\section{Nonlinear Simulations}

\subsection{Resonant Excitation by a Single Galaxy}

We first explore the responses of the adiabatic gas to a single galaxy moving either on a radial or a circular trajectory. BS showed that gravity waves excited by a galaxy with orbital frequency $\omega_{\text {orb }}$ become trapped and amplify in the region where the local BruntVäisälä frequency $\omega_{\mathrm{BV}}$ exceeds $\omega_{\text {orb }}$. This finding was subsequently confirmed by LBH who ran numerical simulations for a radial-orbit galaxy. Our aim here is to find the dependency of energy injection rate on the galaxy mass as well as on the shape of its orbit.

Figure 1a shows the radial distributions of the radial orbit, circular orbit, and BruntVäisälä frequencies in our ICM model. Even without a central massive galaxy, $\omega_{\mathrm{BV}}$ remains almost flat inside $80 \mathrm{kpc}$, allowing resonant excitation of gravity waves there. For models with a radial orbit, the galaxy initially set to move with velocity $v=1740 \mathrm{~km} \mathrm{~s}^{-1}$ from the origin has an average speed of $820 \mathrm{~km} \mathrm{~s}^{-1}$ until it reaches a turning point at $r=150 \mathrm{kpc}$ (black dot in Fig. 1a). For circular-orbit models, the orbital speed is $v=1060 \mathrm{~km} \mathrm{~s}^{-1}$ at $r=130 \mathrm{kpc}$ (blue dot). In both cases, the orbital period of the galaxy is $0.73 \mathrm{Gyr}$, which is chosen to ensure $\omega_{\mathrm{BV}}>\omega_{\text {orb }}$ inside $r_{c}$.

As the galaxy starts to move, it generates density and velocity perturbations, forming a gravitational wake and imparting some of its gravitational energy to thermal and kinetic energies of the ICM. In general, the perturbations are a superposition of $p$ - and $g$-waves. While high-frequency $p$-waves propagate out through a stratified background, outgoing $g$ waves reflected at, and remain trapped within, the resonance radius ( $\sim r_{c}$ in our models). The gas near the center receives periodic kicks from the galaxy, enhancing the levels of density and velocity fluctuations. Overall evolution of the models with a galaxy on the radial orbit is similar to that presented in $\mathrm{LBH}$.

Figure 1 $\mathrm{b}$ shows a snapshot of the perturbed density in the orbital plane at $t=6 \mathrm{Gyr}$

\footnotetext{
${ }^{2}$ We have also run models with $128^{3}$ zones, and checked that the results are within less than $10 \%$ of those from $256^{3}$ runs. This suggests the energy dissipation caused by numerical diffusion is tolerable. See $₫ 3.2$.
} 
for a model where a galaxy with mass $M_{11} \equiv M_{g} /\left(10^{11} \mathrm{M}_{\odot}\right)=5$ orbits circularly at a near transonic speed (Mach number $=0.97$ ) in the clockwise direction. It is apparent that perturbations periodically provided by the orbiting galaxy is focused to the central part. The associated vorticity is also well contained inside the resonance marked by a dotted circle, indicative of resonant excitation (LHB). The density perturbations near the galaxy in a weak trailing shape are a characteristic feature of a wake for a circular-orbit perturber (Kim \& Kim 2007).

As the galaxy orbits the cluster center and resonantly interacts with the background gas, the kinetic energy absorbed in the ICM secularly increases (approximately linearly) with time, while showing some temporal fluctuations. We run a number of models with varying $M_{g}$, and measure the rate $\dot{E}_{k}$ at which kinetic energy increases inside $r_{c}$. Figure 1. plots the resulting $\dot{E}_{k}$, which are fairly well fitted by $\dot{E}_{k}=1.2 \times 10^{40} M_{11}^{2} \mathrm{erg} \mathrm{s}^{-1}$ and $\dot{E}_{k}=3.5 \times 10^{39} M_{11}^{2} \mathrm{erg} \mathrm{s}^{-1}$ for the radial- and circular-orbit cases, respectively. For the model parameters we adopt, therefore, a galaxy on a radial orbit is about three times more efficient in driving kinetic energy into the ICM than the circular-orbit counterpart, since it in the former orbit can traverse the central region directly (BS). The dependency of $\dot{E}_{k}$ on $M_{g}^{2}$ indicates that perturbations in all the models with a single galaxy are in the linear regime.

\subsection{Heating by Cluster Galaxies}

We now consider more realistic cluster models in which the ICM is continuously stirred by many member galaxies. We run nine adiabatic models as well as nine cooling counterparts. Figure 2 plots time evolution of the kinetic energy $E_{k}$ of the ICM inside $r_{c}$ for some of the adiabatic models. A sudden introduction of the galaxies causes the ICM to respond abruptly, initiating an rapid increase of $E_{k}$ for $t<0.1$ Gyr. For models D $\left(N_{g}=10^{2}\right.$, $\left.M_{11}=5\right)$ and $\mathrm{E}\left(N_{g}=10^{2}, M_{11}=1\right)$, the perturbations are initially in the linear regime and soon begin to interact resonantly with the background gas. As gravity waves concentrate toward the center and amplify, $E_{k}$ grows secularly with time. For $t>2$ Gyr, $E_{k}$ in models $\mathrm{D}$ and $\mathrm{E}$ (and other models with $N_{g}^{1 / 2} M_{11} \lesssim 100$ ) increases almost linearly at a rate $\dot{E}_{k}=$ $2.6 \times 10^{41}\left(N_{g} / 10^{2}\right) M_{11}^{2} \mathrm{erg} \mathrm{s}^{-1}$. Compared to the results of $\$ 3.1$, this corresponds to about 20 radial-orbit galaxies taking part in resonant excitation of the ICM inside $r_{c}$. On the other hand, in model $\mathrm{A}\left(N_{g}=10^{3}, M_{11}=10\right)$ where initial perturbations are very strong, $E_{k}$ stays almost constant at $\sim 10^{60.5} \mathrm{erg}$ during its entire evolution, indicating weak or no resonant excitation. Models $\mathrm{B}\left(N_{g}=10^{3}, M_{11}=5\right)$ and $\mathrm{C}\left(N_{g}=500, M_{11}=5\right)$ that have weaker initial perturbations than model A enhance $E_{k}$ to the saturation level $\sim 10^{60.5}$ erg for $t \lesssim 1$ Gyr, after which $E_{k}$ again remains constant. At saturation, the amplitudes of density 
fluctuations are about $40 \%$ relative to the mean value, easily disrupting galaxy wakes that would otherwise supply fresh perturbations for resonant excitation. Consequently, resonant excitation becomes weak or even absent in a highly nonlinear background.

Unlike kinetic energy that grows with time when $N_{g} M_{g}^{2}$ is sufficiently small, we find that thermal energy of the ICM does not show a clear indication of resonant excitation. The total amount of thermal energy within $r_{c}$ driven by a mere introduction of the galaxies is $\Delta E_{t}=10^{58} N_{g} M_{11} \mathrm{erg}$, which is already large and stays more or less constant with time for all the models. This may be because kinetic energy is more prone to resonant excitation, or because the amount of heat supplied (presumably at a rate similar to $\dot{E}_{k}$ ) by resonant excitation is much smaller than $\Delta E_{t}$, so that it does not readily manifest in the energy curves over time. The ratio of kinetic to thermal energies in a wake produced by a single galaxy with size $a$ and velocity $\sigma_{v}$ is roughly $\sim\left(G M_{g} / \sigma_{v} a\right)^{2} /\left(3 c_{s}^{2}\right)$, where $c_{s}$ is the adiabatic sound speed of the ICM (Just \& Kegel 1990). Since this ratio is less than $10^{-2}$ when $M_{11} \leq 1$ for typical parameters we adopt, kinetic energy is certainly a much better tracer of resonant excitation.

To check if galaxy motions and the associated heating can solve the cooling flow problem, we repeat the simulations by including the radiative cooling explicitly. Our models lose thermal energy at a rate $L_{X} \sim 10^{44} \mathrm{erg} \mathrm{s}^{-1}$ from the cooling core. Without any heat source, they would experience a cooling catastrophe within $0.6 \mathrm{Gyr}$. It turned out that none of the models we considered were able to prevent, albeit considerably delay, the runaway cooling. For instance, the cooling model with $N_{g}=10^{2}$ and $M_{11}=5$ (corresponding to model D) undergoes a catastrophic event at $2.5 \mathrm{Gyr}$, while the model $N_{g}=10^{3}$ and $M_{11}=10$ (corresponding to model A) develops a cooling flow in 1.7 Gyr near the center. The heating rate due to resonant excitation is probably lower in the latter model, for which the perturbed kinetic energy saturates immediately.

\subsection{Properties of ICM Turbulence}

We have seen that motions of the member galaxies generate a large amount of kinetic energy in the ICM. The kinetic energy is in the form of fluctuating isotropic velocity fields with vanishingly small mean values. We regard these spatially-uncorrelated, random gas motions as ICM turbulence. To quantify the turbulence level, for each run we measure the velocity dispersions of the gas inside $r_{c}$. Figure 3 a plots as open circles the resulting density-weighted $3 \mathrm{D}$ velocity dispersion $\sigma_{v} \equiv\left(2 E_{k} / M_{c}\right)^{1 / 2}$ averaged over $3-6$ Gyr as a function of $N_{g} M_{11}^{2}$, where $M_{c}$ is the ICM mass inside $r_{c}$. Filled circles give non-weighed velocity dispersions, which is larger than the density-weighted values by about a factor of 
1.5. Consistent with the results of the previous subsection, $\sigma_{v}$ increases linearly with $N_{g}^{1 / 2} M_{11}$ until $N_{g}^{1 / 2} M_{11} \approx 100$, beyond which $\sigma_{v}$ is approximately constant at $\sim 210-230 \mathrm{~km} \mathrm{~s}^{-1}$.

To characterize the turbulence driven by galaxy motions, we calculate Fourier power spectra of the compressive and shear components of ICM velocities defined by $v_{c}^{2}=\left|\hat{\mathbf{k}} \cdot \mathbf{v}_{k}\right|^{2}$ and $v_{s}^{2}=\left|\hat{\mathbf{k}} \times \mathbf{v}_{k}\right|^{2}$, respectively. Here, $\mathbf{v}_{k}$ is the Fourier transformed velocity and $\hat{\mathbf{k}}$ is the wavenumber. We then bin them spherically in the $\hat{\mathbf{k}}$-space and calculate the angle-averaged power spectra $P_{c}\left(k_{r}\right)$ and $P_{s}\left(k_{r}\right)$ as functions of the radial wavenumber $k_{r}$. Figure $3 \mathrm{~b}$ shows $P_{c}$ and $P_{s}$ for models $\mathrm{C}$ and $\mathrm{E}$ at $t=6 \mathrm{Gyr}$. The ratio of total power in the compressive to shearing parts is about 2.5 for both models, indicative of fairly subsonic turbulence (e.g., Vestuto \& Ostriker 2003). The power index of the shearing part is -3.7 in the inertial range for model $\mathrm{E}$, which becomes steeper with increasing $\sigma_{v}$, yielding -4.3 for model $\mathrm{d} 3$. The compressive parts have similar slopes, although they have excess power at $\sim 50-100 \mathrm{kpc}$ scales, which appear to be associated with the mean galaxy separations 4 .

\section{Discussion}

Galaxies in a cluster contain a plentiful amount of energy in their orbital motions that can be transferred to turbulent kinetic and thermal energies of the ICM (e.g., BS; Deiss \& Just 1996). In this Letter, we have shown that resonant excitation of gravity waves driven by galaxy motions is efficient only when density and velocity fluctuations in the background are in the linear regime, while becoming inefficient when the background medium exhibits large amplitude fluctuations. Although it is uncertain at what rate resonant excitation heats the ICM, our numerical results suggest that heating by galaxy motions is insufficient to quench the cooling catastrophe. If the heating rate is similar to the kinetic energy injection rate $\dot{E}_{k}$ we found, the energy balance between the heating and X-ray cooling requires $N_{g}^{1 / 2} M_{11} \sim 200$. Although rich clusters may contain enough number and mass of galaxies to satisfy this condition, resonant excitation will switch off when this condition is met. We thus conclude that heating by galaxy motions alone cannot be the main solution to the cooling flow problem, although it can delay the catastrophic event significantly (e.g., BS; Kim et al 2005).

The two key parameters that control the rate of energy injection and the level of turbu-

\footnotetext{
${ }^{3}$ Caution should be made in interpreting the power indices in comparison with Kolmogorov power spectra, since in our models the background density is not uniform and the velocity field is not periodic.

${ }^{4}$ The galaxies are concentrated more strongly toward the center and have a mean distance of $82 \mathrm{kpc}$ inside the cooling radius.
} 
lence are the mass and number of galaxies. Many uncertainties surround the observational determinations of the average galaxy mass, but a recent analysis using strong-lensing models shows that a cluster galaxy can have mass as large as $5 \times 10^{11} \mathrm{M}_{\odot}$ including a dark halo (Halkola et al. 2007). For rich clusters with $N_{g}>10^{3}$ inside $1 \mathrm{Mpc}$, therefore, our numerical results suggest that ICM turbulence driven solely by galaxy motions is probably in a saturated state with $\sigma_{v} \sim 220 \mathrm{~km} \mathrm{~s}^{-1}$. Given the many arbitrary choices for the cluster parameters, this value is in rough agreement with an analytic estimate of $\sim 300 \mathrm{~km} \mathrm{~s}^{-1}$ by Subramanian et al (2006) who used scaling laws of hydrodynamic wakes without considering nonlinear saturation. Note that the saturated $\sigma_{v}$ is similar to those required to explain the observed magnetic field strength $(\sim 1 \mu \mathrm{G})$ in terms of energy equipartition (e.g., Goldman \& Rephaeli 1991).

The angle-averaged velocity power spectra of turbulence driven in our models are characterized by inertial-range slopes ranging from -3.7 to -4.3 . It is interesting to note that these are comparable to the values between $-11 / 3$ and $-13 / 3$ inferred from the observed pressure maps of the ICM in the Coma cluster (Schuecker et al. 2004), although density inhomogeneities created by recent infall/mergers (e.g., Adami et al 2005) are likely to influence the pressure maps of the Coma cluster that is dynamically young. Obviously, there are other potential driving sources including AGN and subcluster mergers, ram pressure stripping, etc. It will be interesting to see how turbulence driven by each process adds together when nonlinear effects as well as magnetic fields are considered.

The author is grateful to an anonymous referee for a helpful report. This work was supported by Korea Science and Engineering Foundation (KOSEF) grant R01-2004-00010490-0. The numerical computations presented in this work were performed on the Linux cluster at KASI built with funding from KASI and ARCSEC.

\section{REFERENCES}

Adami, C., Biviano, A., Durret, F., \& Mazure, A. 2005, A\&A, 443, 17

Balbus S. A., Soker N., 1990, ApJ, 357, 353 (BS)

Bregman, J. N., \& David, L. P. 1989, ApJ, 341, 49

Clarke, T. E., Kronberg, P. P., \& Böhringer, H. 2001, ApJ, 547, L111

Deiss, B. M., \& Just, A. 1996, MNRAS, 305, 407 
Dennis, J. T., \& Chandran, B. D. G. 2005, ApJ, 622, 205

El-Zant, A., Kim, W.-T., \& Kamionkowski, M. 2004, MNRAS, 354, 169

Fujita, Y., \& Reiprich, T. H. 2004, ApJ, 612, 9

Gerhard, O., Kronawitter, A., Saglia, R. P., \& Bender, R. 2001, AJ, 121, 1936

Goldman, I., \& Rephaeli, Y. 1991, ApJ, 380, 344

Halkola, A., Seitz, S., \& Pannella, M. 2007, ApJ, 656, 739 ApJ, 656, 739

Just, A., \& Kegel, W. H. 1990, A\&A, 232, 447

Kim, W.-T., \& Narayan R. 2003, ApJ, 596, L139

Kim, W.-T., El-Zant, A. A., Kamionkowski, M. 2005, ApJ, 632, 157

Kim, H., \& Kim, W.-T. 2007, ApJ, in press; astro-ph/0705.0084

Lufkin, E. A., Balbus, S. A., \& Hawley, J. F. 1995, ApJ, 446, 529 (LBH)

Miller, L. 1986, MNRAS, 220, 713

Navarro, J. F., Frenk, C. S., \& White, S. D. M. 1997, ApJ, 490, 493

Ostriker, E. C. 1999, ApJ, 513, 252

Peterson, J. R., \& Fabian, A. C. 2006, Physics Reports, 427, 1

Ruszkowski, M., \& Begelman, M. C. 2002, ApJ, 581, 223

Schuecker, P., Finoguenov, A., Miniati, F., Böhringer, H., \& Briel, U. G. 2004, A\&A, 426, 387

Stone, J. M., \& Norman, M. L. 1992, ApJS, 80, 753

Subramanian, K., Shukurov, A., \& Haugen, N. E. L. 2006, MNRAS, 366, 1437

Vernaleo, J. C., Reynolds, C. S. 2006, ApJ, 645, 83

Vestuto, J. G., \& Ostriker, E. C. 2003, ApJ, 590, 858

Voigt, L., \& Fabian, A. MNRAS, 347, 1130 

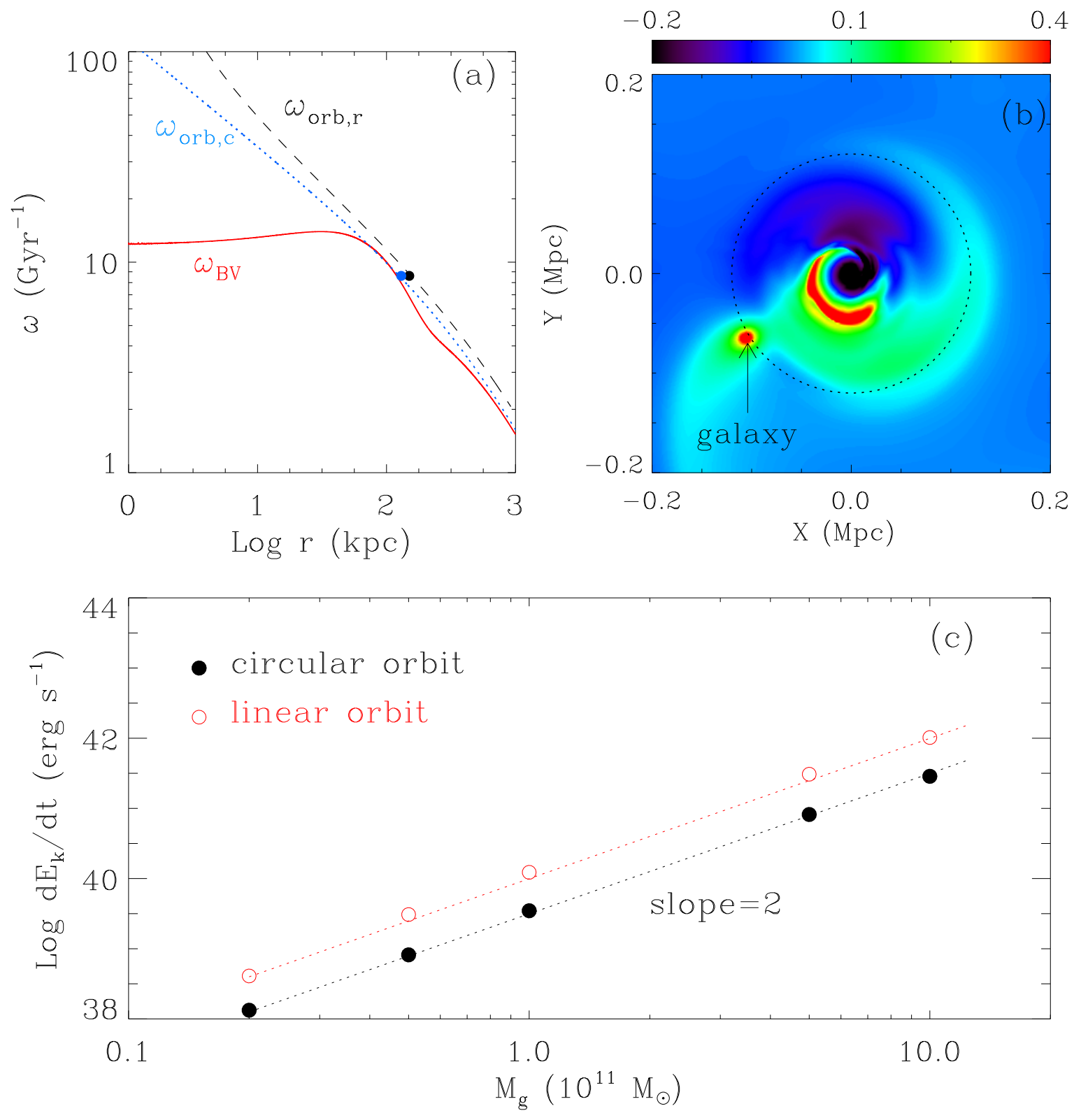

Fig. 1.- (a) Profiles of the radial orbit $\left(\omega_{\text {orb,r }}\right)$, circular orbit $\left(\omega_{\text {orb,c }}\right)$, and Brunt-Väisälä $\left(\omega_{\mathrm{BV}}\right)$ frequencies in our cluster model. (b) $X-y$ plane snapshot at $t=6 \mathrm{Gyr}$ of the perturbed density relative to the initial value in logarithmic scale for a circular-orbit galaxy. (c) Averaged input rates of the kinetic energy due to a single galaxy as functions of its mass. 




Fig. 2.- Temporal evolution of the kinetic energy $E_{k}$ inside the cooling radius for adiabatic models that differ in the number and mass of the galaxies. Solid curves show the results from runs with $256^{3}$ zones. Dotted lines (for models D and E) from $128^{3}$-zone runs are within $8 \%$ of the corrresponding solid curves. Dashed and dot-dashed lines draw $E_{k}=$ $8.2 \times 10^{57} M_{11}^{2}(t / \mathrm{Gyr})$ erg for $M_{11}=1$ and 5 , respectively. 

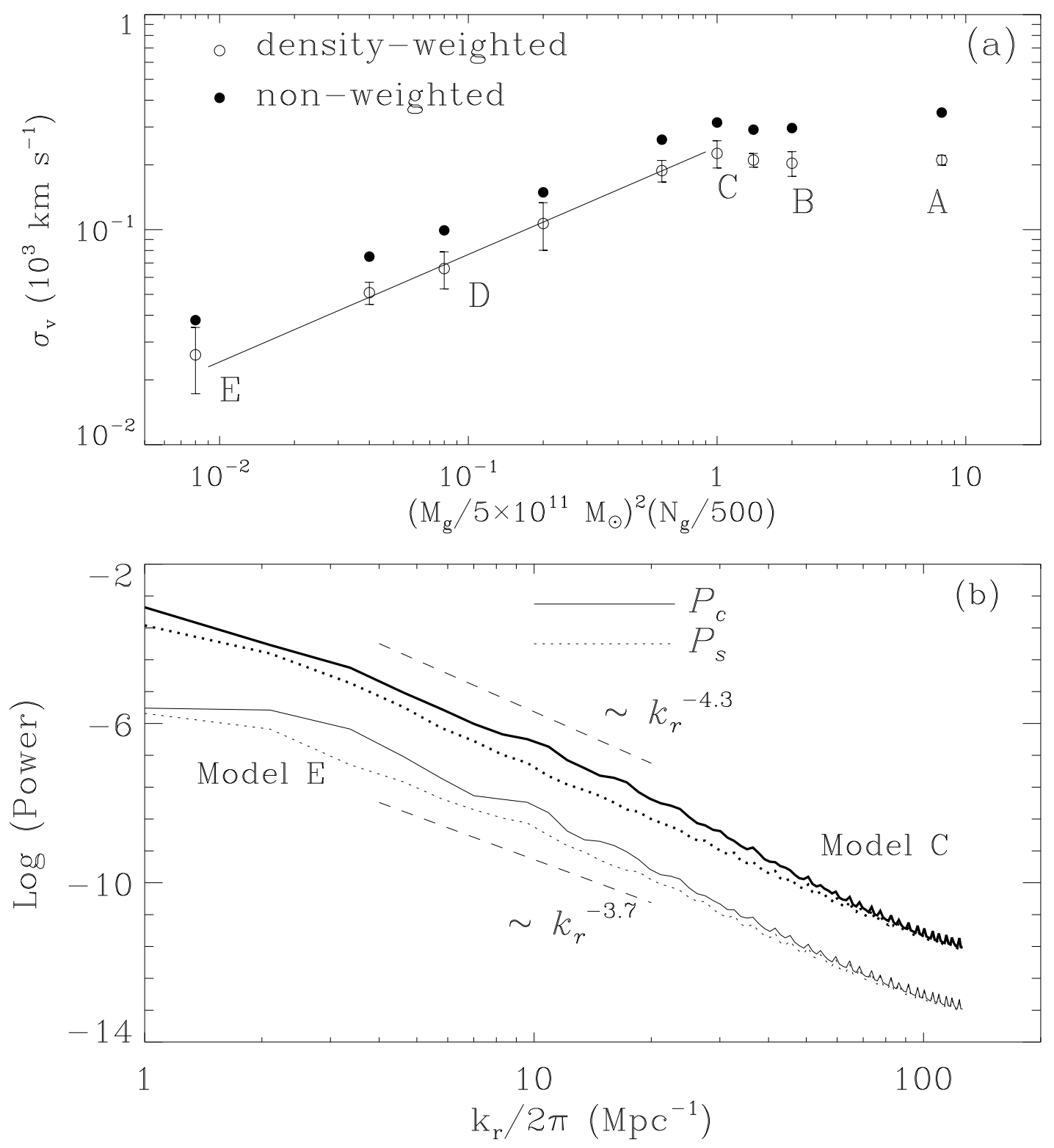

Fig. 3.- (a) Three-dimensional velocity dispersions $\sigma_{v}$ of the ICM turbulence driven by galaxy motions as functions of $N_{g} M_{11}^{2}$. Open circles, with errorbars representing the standard deviations in the temporal fluctuations of $\sigma_{v}$, denote the density-weighted values, while the non-weighted ones are given by filled circles. The solid line corresponds to $\sigma_{v}=2.2 \mathrm{~km} \mathrm{~s}^{-1} N_{g}^{1 / 2} M_{11}$. (b) Spherically binned power spectra of (solid line) compressive and (dotted line) shearing parts of the ICM velocity in models $\mathrm{C}$ and $\mathrm{E}$ at $t=6 \mathrm{Gyr}$. 